\title{
Association of HIVEP3 Gene and Lnc RNA with Femoral Neck Bone Mineral Content and Hip Geometry by Genome-Wide Association Analysis in Chinese People
}

\author{
Weiwei Hu, ${ }^{1}$ Jinwei He, ${ }^{1}$ Luyue Qi, ${ }^{1}$ Chun Wang, ${ }^{1}$ Hua Yue, ${ }^{1}$ Jiemei Gu, ${ }^{1}$ Hao Zhang, \\ Yi Wang $\mathbb{C}^{2,3}$ and Zhenlin Zhang $\mathbb{\circledR}^{1}$ \\ ${ }^{1}$ Shanghai Clinical Research Center of Bone Diseases, Department of Osteoporosis and Bone Diseases, \\ Shanghai Jiaotong University Affiliated Sixth People's Hospital, Yishan Road 600, Shanghai 200233, China \\ ${ }^{2}$ Ministry of Education Key Laboratory of Contemporary Anthropology, \\ Collaborative Innovation Center for Genetics and Development, School of Life Sciences, Shanghai, China \\ ${ }^{3}$ Human Phenome Institute, Fudan University, Shanghai, China \\ Correspondence should be addressed to Yi Wang; godspeed_china@yeah.net and Zhenlin Zhang; zhangzl@sjtu.edu.cn
}

Received 8 April 2020; Revised 27 July 2020; Accepted 30 September 2020; Published 13 October 2020

Academic Editor: Christian S. G bl

Copyright $\odot 2020$ Weiwei Hu et al. This is an open access article distributed under the Creative Commons Attribution License, which permits unrestricted use, distribution, and reproduction in any medium, provided the original work is properly cited.

\begin{abstract}
Purpose. GWAS has successfully located and analyzed the pathogenic genes of osteoporosis. Genetic studies have found that heritability of BMD is $50 \%-85 \%$ while the other half is caused by hip geometric parameters and tissue horizontal characteristics. This study was designed to study the GWAS of osteoporosis in Shanghai Han population. Methods. We collected 1224 unrelated healthy young men (20-40 years old), young women (20-40 years old), and postmenopausal women (over 50 years old) who lived in Shanghai. BMD and hip geometric parameters were measured by dual-energy X-ray absorptiometry. The genomic DNA of peripheral blood was extracted and analyzed by using Illumina Human Asian Screening Array-24 + v1.0 (ASA) gene chip. Statistical analysis was carried out to evaluate the relationship between these SNPs and BMD and hip geometric parameters. Results. A total of 1155 subjects were included. We found that one SNP rs35282355 located in the human immunodeficiency virus type 1 enhancer-binding protein 3 gene (HIVEP3) and another 25 SNPs located in LINC RNA were significantly correlated with bone mineral content $(\mathrm{BMC})$ in the femoral neck $\left(P=2.30 \times 10^{-9}, P<5 \times 10^{-8}\right)$. We also found that the correlation between SNP rs35282355 and cross-sectional area (CSA) of hip geometry was a significant marginal statistical difference $\left(P=5.95 \times 10^{-8}\right)$. Conclusions. Through this study, we found that HIVEP3 gene and LINC RNA are potentially correlated with femoral neck BMC. These results provide important information for us to further understand the etiology and genetic pathogenesis of osteoporosis. In the future, we will expand the sample size to verify these loci and carry out molecular research.
\end{abstract}

\section{Introduction}

Osteoporotic fracture (OF) is the most severe clinical outcome of osteoporosis, which confers substantial morbidity, mortality, and social services expenditure in the elderly $[1,2]$. At present, in the world, there have been more than 9 million fractures annually due to osteoporosis, of which 1.6 million were at the hip, 1.7 million were at the forearm, and 1.4 million were clinical vertebral fractures $[1,3]$. In China, there was an obvious increase in the prevalence of osteoporosis from $14.94 \%$ before 2008 to $27.96 \%$ during the period spanning 2012-2015; this brings an enormous economic burden on the whole society [4]. Our study showed that the prevalence of vertebral fracture was $17.2 \%$ among Shanghai community-dwelling elders (over 60 years), 17.0\% for males, and $17.3 \%$ for females. The prevalence among females increased with age [5]. The prevalence of osteoporosis in our country has increased over the past 12 years, affecting more than one-third of people aged 50 years and older [4].

Measurement of bone mineral density (BMD) has been used for the gold standard for the diagnosis of osteoporosis 
and the assessment of fracture risk. Variations in BMD can be explained by genetic effects, with which heritability ranges from 0.5 to $0.8[6,7]$. To date, there are more than 100 candidate genes/loci associated with variations in BMD which have been identified in genome-wide association studies (GWASs) and their meta-analyses [8, 9]. Many countries performed GWAS and osteoporosis: in UK, they identified 203 single-nucleotide polymorphisms (SNPs) associated with BMD as estimated by quantitative ultrasound of the heel by GWAS in 142,487 individuals [10]. Icelandic deCODE Study (dCG) discovered multiple genetic loci associated with spine and femoral neck BMD [11]. The first multiethnic BMD GWAS used the Europeans as a discovery population, also the Chinese population and the African people in this study. This particular GWAS identified ADAMTS18 and TGFBR3 as bone mass candidate genes in different ethnic groups [9]. But in this study, there were only a Chinese hip fracture sample and 2955 Chinese BMD subjects for replication. We found that GWAS and BMD in China began in 2010, only a Chinese hip fracture sample (350 with hip OF and 350 healthy matched controls) [12]. After that, Tan et al. published GWAS and osteoporosis again and found ATP6V1G1 as a novel locus underlying osteoporosis and age at menarche in 2015 [13]. In Hong Kong, a GWAS about 800 unrelated Chinese women with extremely high or low BMD was performed and the JAG1 gene was identified as a candidate for BMD regulation in 2010 [14]. So far, the data of BMD and GWAS of Han nationality in China are very few. Through PubMed search, we found that most of the current studies on BMD and GWAS in China were based on these two data sets $[12,14]$.

However, BMD does not accurately describe either the strength or a specific geometric configuration of bone tissue [15]. Our previous study also proved that hip geometry parameters are risk factors for hip fracture and that these are independent of BMD measurements [16]. Up to now, there were a few studies which performed GWAS on hip geometry measures [17]. By GWAS, femoral neck-shaft angle (NSA) was found for SNPs located on chromosome 2q11.2 (TBC1D8) in EU populations [17]. Another GWAS study in 1000 Caucasians found that rs7430431, in the receptor transporting protein 3 (RTP3) gene, was identified in strong association with hip geometry, the buckling ratio (BR), and femoral cortical thickness (CT), an index of bone structural instability [18]. By referring to the PubMed literature, there were only few papers on GWAS and hip geometry in the world, and there was no large sample about GWAS and hip geometry about Chinese Han people.

To identify genetic variants that influence BMD in different ethnic groups, we performed a GWAS on 1224 unrelated Chinese women and men in Shanghai with BMD parameters. In the present study, we also aimed to report the GWAS of hip geometry of these subjects, which is thought to be related to the risk of hip fracture, identified BMD-associated genes. Our study also was used as input information for subsequent gene enrichment analysis to assess their potential biological roles in osteoporosis pathophysiology.

\section{Materials and Methods}

2.1. Subjects. In total, 1224 unrelated subjects were collected, including 366 young men aged 20-40 years, 239 young premenopausal women aged 20-40 years, and 619 postmenopausal women aged 50-80 years. All subjects were Chinese Han people selected for inclusion in the study. The inclusion criteria were as follows: healthy men and women according to the following excluding criteria. Exclusion criteria in accordance with our previous articles were used to exclude individuals from the study $[5,16,19,20]$ : (1) serious effects from cerebrovascular disease; (2) other diseases, such as diabetes mellitus, chronic renal disease, chronic liver disease, rheumatoid arthritis or collagen disease, hyperthyroidism, and recent major gastrointestinal disease; (3) evidence of other metabolic or inherited bone diseases; (4) significant diseases of any endocrine organ that would affect bone mass; (5) any neurological or musculoskeletal condition; and (6) any form of calcium and vitamin-D therapy in three months or taking antiosteoporotic drugs (e.g., bisphosphonates, selective estrogen receptor modulators, and calcitonin). Postmenopausal women who had experienced early menopause (before 45 years of age) were excluded. All study subjects belonged to the Chinese Han ethnic group. All were residents of Shanghai City, located in the mideastern coast of China. The study was approved by the ethics committee of the Shanghai Jiao Tong University Affiliated Sixth People's Hospital.

\subsection{BMD and Hip Geometry Parameters Measurements.} The BMD of the lumbar spine 1-4 (L1-4), the left proximal femur, bone mineral content (BMC), and hip geometry parameters were measured by DXA on a GE-LUNAR Prodigy (Lunar Corp., Madison, WI). All hip scans were reprocessed using enCore Installation 13.10.078 software (GE Healthcare). As our previous study described, this software includes advanced hip analysis, which provides the following structural parameters: hip axis length (HAL), cross-sectional moment of inertia (CSMI), cross-sectional area (CSA), and femoral strength index (SI) [20]. The root mean square coefficient of variability (RMSCV\%) values of $\mathrm{BMD}$ and hip geometry were obtained from our previous studies. The RMSCV\% values of the DXA measurements at L1-4, the femoral neck, trochanter, and total hip were $1.39 \%$, $2.22 \%, 1.41 \%$, and $0.70 \%$, respectively [16]. The RMSCV\% values of the HAL, CSMI, CSA, and SI were $1.09 \%$, $4.91 \%$, $2.49 \%$, and $6.07 \%$, respectively $[16,19,20]$.

2.3. Genotyping. High-throughput genome-wide SNP genotyping was performed the Illumina Infinium Asian Screening Array-24+v1.0 BeadChip technology containing 846000 SNPs and following the manufacturer's protocol.

2.4. Statistical Analysis. The data are given as the mean \pm SD for normal data. We divided the data into three groups according to age, and differences between groups were assessed using an ANOVA test with Fisher's protected least 
significant difference post hoc test for normal data. The threshold for the replication significance was set at $P<0.05$. Statistical analyses were performed using SPSS version 21.0 for Windows (SPSS Inc.).

2.5. Phenotype Normalization. Phenotypes were firstly ranktransformed in order to attenuate the impact of outliers and nonnormal distributions. A random forest regression is performed using each phenotype rank as a target while using sex and age as predictors. The out-of-bag estimates (age-sex expected phenotype ranks) are subtracted from the phenotype ranks. Again, this age-sex adjusted rank is ranktransformed as the final phenotype rank and used for later analysis.

2.6. Genotype Imputation. Genotype imputation is performed by a commercial imputation engine named GenoImpute. We obtain a mean sample-level $r^{2}$ of 0.736 estimated by $1 \%$ holdout SNPs on the array. Different from other off-the-shelf imputation engines, this engine produces a continuous allele dosage as well as three-genotype probability distribution which reflects the reality of genotype uncertainty. The allele dosage is used for later analysis.

2.7. Phenotype Rank: SNP Association Test. We employ the Spearman correlation test with a permutation-based exact $P$ value to test phenotype rank-SNP associations. Up to 1010 simulation is performed. According to the statistical analysis of the data mapping analysis: the whole genome trend chisquare test results in SNPs, chromosome, physical location, and $P$ value of the input $R$ software to generate a genomewide association analysis of the Manhattan map and Quantile-Quantile plots map (Q-Q map).

\section{Results}

3.1. Basic Characteristics of the Study Subjects. Figure 1 shows the flow diagram of this study. We obtain 1167 raw chip samples. By filtering samples with low heterozygosity $(<0.165$ according to visual outlier inspection $)$ and a high missing rate $(>0.05)$, we retained 1155 samples. There are 570378 SNPs on the chip. We filtered SNPs with MAF $<0.01$ or $\mathrm{p}-\mathrm{HWE}<0.01$ or miss rate $>0.1$ and retained 474773 SNPs (Figure 2).

The basic characteristics of the samples are summarized in Table 1. BMD of the lumbar spine, femoral neck, and total hip in postmenopausal women were $0.980 \pm 0.152 \mathrm{~g} / \mathrm{cm}^{2}$, $0.784 \pm 0.126 \mathrm{~g} / \mathrm{cm}^{2}$, and $0.846 \pm 0.131 \mathrm{~g} / \mathrm{cm}^{2}$, respectively. Hip and spine BMD or BMC were lower in postmenopausal women than in young men and premenopausal young women. This significant difference observed here is consistent with the previous findings. In the young male group, the hip geometric parameters HAL, CSMI, CSA, and SI were $110.82 \pm 6.40 \mathrm{~mm}, 14034 \pm 3693 \mathrm{~mm}^{4}, 164.49 \pm 26.29 \mathrm{~mm}^{2}$, and $1.59 \pm 0.13$, respectively. In the young men group, there were higher HAL, CSMI, and CSA than the other two groups.
3.2. Relationship between BMC and GWAS, Hip Geometric Parameters, and GWAS. Our quantitative trait analyses identified SNPs by a genome-wide significant threshold $\left(P<5 \times 10^{-8}\right)$. Table 2 shows the distribution of $P$ values of GWASs across the genome in our Chinese samples. We found some significant SNPs with $P<5 \times 10^{-8}$. We listed some SNPs for CSA in Table 2. There is a marginal difference between SNP rs35282355 $\left(P=5.95 \times 10^{-8}\right)$ located in human immunodeficiency virus type 1 enhancer-binding protein 3 (HIVEP3) gene and CSA, suggesting that it is a susceptible site of CSA (Figure 3).

In the femoral neck BMC phenotype, 26 SNPs (see Table 3) reached the genome-wide significance level $\left(P<5 \times 10^{-8}\right)$ (Table 3). Interestingly, the strongest association was found at SNP rs35282355 located in the intron of the HIVEP3 gene, with $P=2.30 \times 10^{-9}$ for neck BMC (Figure 4), which has not been implicated in GWAS of BMD previously. This locus was most strongly associated with neck BMC. While the other 25 SNPs with significant statistical significance were located in the long intergenic nonprotein coding RNA (LINC RNA).

\section{Discussion}

In this study, we performed a GWAS of lumbar spine BMD, proximal femur BMD, lumbar spine BMC, proximal femur $\mathrm{BMC}$, and hip geometry parameters in 1155 participants from Shanghai. The GWAS study of osteoporosis was carried out for the first time in the Han population in Shanghai. We found a SNP rs352355 $\left(P=5.95 \times 10^{-8}\right)$ of HIVEP3 gene located on $1 \mathrm{p} 34.2$ was significantly associated with CSA, suggesting that it was the susceptible site of CSA, a geometric parameter of the hip. At the same time, this locus was found to have genome-wide significance with femoral neck BMC $\left(P=2.30 \times 10^{-9}\right)$. We identified rs35282355 in HIVEP3 gene that was associated with both CSA and femur neck BMC.

HIVEP3, also known as Schnurri-3 (SHN3), was a zinc finger protein and an important regulator of bone formation. This gene had 17 exons, of which rs35282355 was located in the intron region. SHN3-deficient mice exhibited osteosclerosis due to increased osteoblast activity and increased bone mass [21]. In osteoblasts, SHN3 was a component of the trimer complex between Runx2 and E3 ubiquitin ligase WWP1. Because WWP1 promoted ubiquitination of Runx2 and protease-dependent degradation, the complex inhibited Runx 2 function and gene expression involved in extracellular matrix mineralization. SHN3 controls protein levels by degrading Runx 2 which was an essential regulator of osteoblastogenesis, the factor that had been validated [21-23]. In 2013, Shim et al. [24] found that $\mathrm{SHN} 3$ is an attenuator of extracellular regulated protein kinases (ERK) activity. In osteoblasts, ERK was the key to signal transduction from surface receptors to nuclei. ERK activity played a role downstream of WNT signal transduction. $D$ domain in SHN3 mediated the interaction and inhibition of ERK activity and osteoblast differentiation [24]. The knock-in of SHN3 gene mutation eliminated this interaction, which led to abnormal ERK activation and excessive activity of osteoblasts in vivo. In addition, the study 


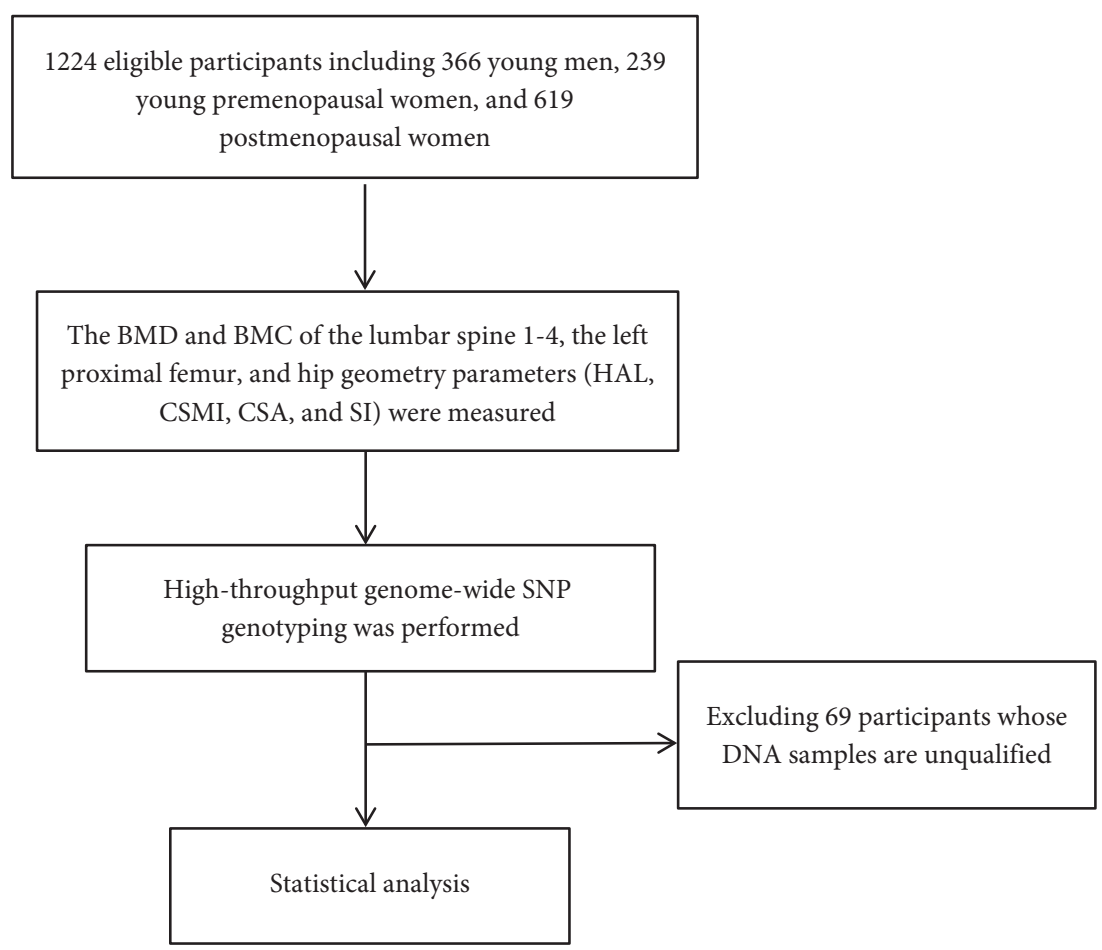

Figure 1: Flow diagram of the study. This is a brief flow chart of this experiment, indicating the number of screening, screening steps, and other details.

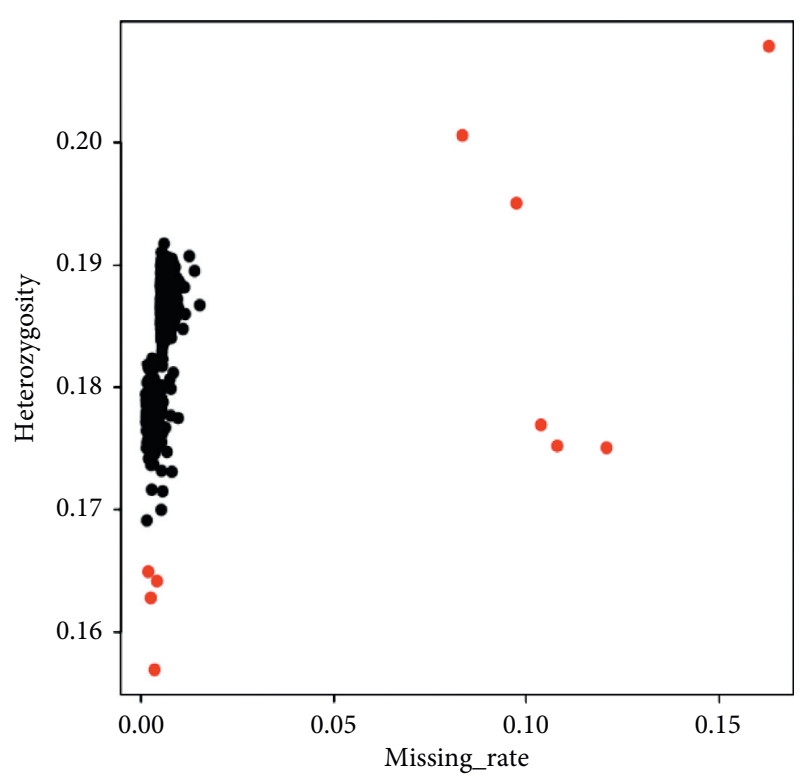

(a)

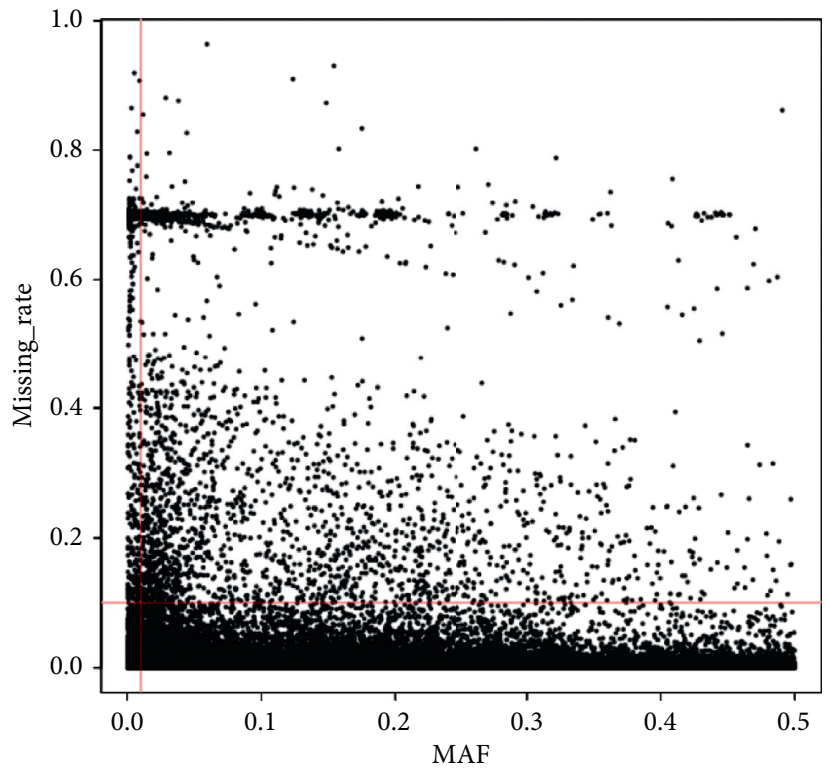

(b)

FIGURE 2: The principal analysis of the Han population samples in Shanghai. There were 570378 SNPs on the chip. SNPs were filtered with $\mathrm{MAF}<0.01$ or $\mathrm{p}-\mathrm{HWE}<0.01$ or miss rate $>0.1$ and retained 474773 SNPs. (a) Sample QC. (b) Marker QC.

also showed that hybridization with $\mathrm{Lrp}^{-/-}$mice partially saved the osteosclerosis phenotype of $\mathrm{SHN}^{-/-}$mice, which corresponded to the ability of SHN3 to inhibit ERK-mediated GSK3 beta inhibition. Induced knockout of Shn3 in adult mice led to high bone mass phenotypes, providing evidence that temporary blockade of these pathways had the potential for the treatment of osteoporosis in adult mice [24]. By searching PubMed, this study was the first to find that the HIVEP3/SHN3 gene had a potential relationship with hip bone mass in the Chinese Han population of GWAS 
TABLE 1: Basic characteristics of the studied samples.

\begin{tabular}{|c|c|c|c|c|}
\hline Traits & Total sample & Young men & Young women & Postmenopausal women \\
\hline $\mathrm{N}$ & 1155 & 341 & 220 & 594 \\
\hline Age (y) & $47.88 \pm 17.85$ & $28.86 \pm 6.12^{*}$ & $32.47 \pm 5.87^{*}$ & $64.28 \pm 7.10^{*}$ \\
\hline Height (m) & $162.0 \pm 9.7$ & $173.0 \pm 6.0^{*}$ & $160.1 \pm 5.3^{*}$ & $154.8 \pm 5.1^{*}$ \\
\hline Weight (kg) & $62.05 \pm 11.06$ & $70.62 \pm 10.20^{*}$ & $55.93 \pm 8.44^{*}$ & $58.67 \pm 8.76^{*}$ \\
\hline Spine BMD $\left(\mathrm{g} / \mathrm{cm}^{2}\right)$ & $1.075 \pm 0.166$ & $1.139 \pm 0.139^{*}$ & $1.172 \pm 0.121^{*}$ & $0.980 \pm 0.152^{*}$ \\
\hline Neck BMD $\left(\mathrm{g} / \mathrm{cm}^{2}\right)$ & $0.887 \pm 0.161$ & $1.003 \pm 0.139^{*}$ & $0.924 \pm 0.112^{*}$ & $0.784 \pm 0.126^{*}$ \\
\hline Total hip BMD $\left(\mathrm{g} / \mathrm{cm}^{2}\right)$ & $0.927 \pm 0.150$ & $1.015 \pm 0.136^{*}$ & $0.959 \pm 0.117^{*}$ & $0.846 \pm 0.131^{*}$ \\
\hline Spine BMC (g) & $58.58 \pm 13.73$ & $69.13 \pm 11.85^{*}$ & $60.96 \pm 9.06^{*}$ & $49.72 \pm 10.24^{*}$ \\
\hline Neck BMC (g) & $4.36 \pm 1.03$ & $5.35 \pm 0.84^{*}$ & $4.36 \pm 0.61^{*}$ & $3.65 \pm 0.62^{*}$ \\
\hline Total hip BMC (g) & $28.17 \pm 5.87$ & $33.49 \pm 4.9^{*}$ & $27.07 \pm 3.78^{*}$ & $24.79 \pm 4.19^{*}$ \\
\hline $\mathrm{HAL}(\mathrm{mm})$ & $102.32 \pm 8.49$ & $110.82 \pm 6.40^{*}$ & $98.25 \pm 5.69$ & $98.17 \pm 6.08$ \\
\hline $\operatorname{CSMI}\left(\mathrm{mm}^{4}\right)$ & $9215 \pm 4192$ & $14034 \pm 3693^{*}$ & $7865 \pm 2677^{*}$ & $6953 \pm 2138^{*}$ \\
\hline $\operatorname{CSA}\left(\mathrm{mm}^{2}\right)$ & $139.47 \pm 236.98$ & $164.49 \pm 26.29^{*}$ & $132.79 \pm 18.97$ & $127.61 \pm 340.99$ \\
\hline SI (dimensionless) & $1.65 \pm 4.23$ & $1.59 \pm 0.13$ & $1.68 \pm 0.48$ & $1.67 \pm 6.09$ \\
\hline
\end{tabular}

Continuous variables expressed as mean \pm SD. ${ }^{*}$ Statistical differences between groups, $P<0.05$.

TABLE 2: The results of CSA associated SNPs in GWAS of the Han population in Shanghai (partially).

\begin{tabular}{|c|c|c|c|c|}
\hline Phenotype & SNP & Alleles & $P$ & Gene \\
\hline CSA & rs35282355 & $G: A$ & $5.95 E-08$ & HIVEP3 \\
\hline CSA & rs7071262 & $\mathrm{T}: \mathrm{G}$ & $7.05 E-07$ & Null \\
\hline CSA & rs7907051 & $\mathrm{G}: \mathrm{A}$ & $2.42 E-07$ & Null \\
\hline CSA & rs187172215 & $\mathrm{T}: \mathrm{C}$ & $8.64 E-07$ & KLHL29 \\
\hline CSA & rs12152026 & $\mathrm{T}: \mathrm{C}$ & $4.99 E-07$ & Null \\
\hline CSA & rs117963651 & $\mathrm{G}: \mathrm{A}$ & $6.48 E-07$ & Null \\
\hline CSA & rs190389312 & $A: G$ & $2.26 E-07$ & Null \\
\hline CSA & rs17592374 & $\mathrm{T}: \mathrm{C}$ & $8.03 E-06$ & Null \\
\hline CSA & rs61791524 & $A: G$ & $7.84 E-06$ & Null \\
\hline CSA & rs61791526 & $T: G$ & $7.84 E-06$ & Null \\
\hline CSA & rs138917572 & $C: T$ & $1.44 E-06$ & Null \\
\hline CSA & rs647297 & $\mathrm{G}: \mathrm{A}$ & $6.99 E-06$ & Null \\
\hline CSA & rs200674613 & $\mathrm{T}: \mathrm{C}$ & $8.30 E-07$ & Null \\
\hline CSA & rs146148517 & $C: G$ & $9.25 E-06$ & Null \\
\hline CSA & rs117331232 & $C: T$ & $9.02 E-06$ & Null \\
\hline CSA & rs7006122 & $\mathrm{T}: \mathrm{G}$ & $6.81 E-06$ & Null \\
\hline CSA & rs5969489 & $\mathrm{G}: \mathrm{A}$ & $4.95 E-06$ & Null \\
\hline CSA & rs200674613 & $\mathrm{T}: \mathrm{C}$ & $8.30 E-07$ & Null \\
\hline
\end{tabular}

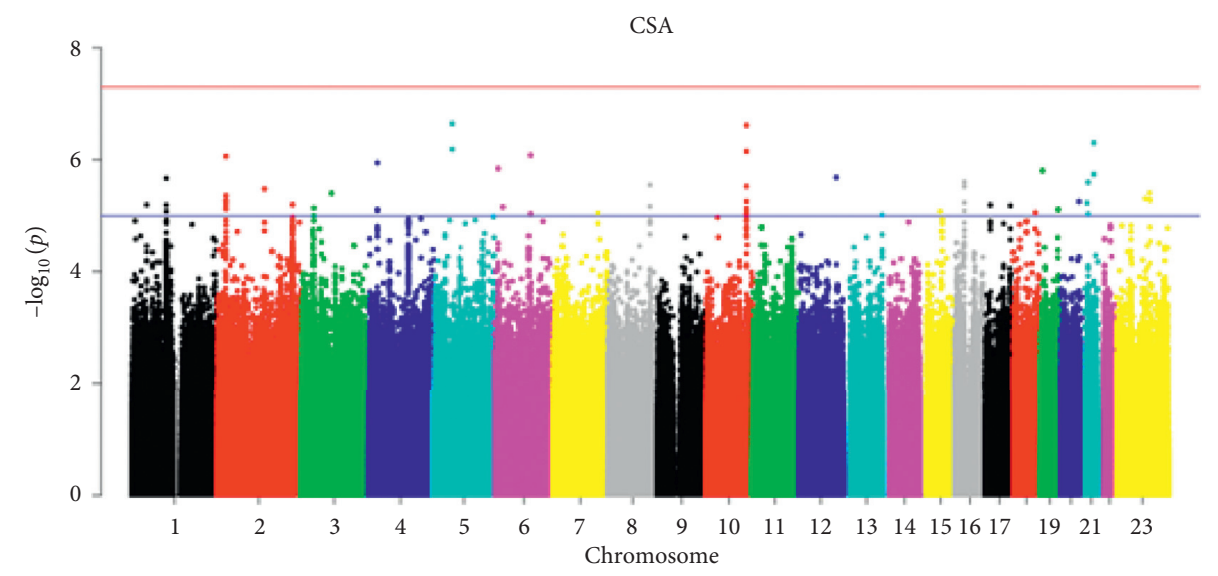

- rs35282355

Figure 3: Manhattan plot of CSA (chromosomal plot). 
TABLE 3: The results of femoral neck BMC associated SNPs in GWAS of the Han population in Shanghai (partially).

\begin{tabular}{|c|c|c|c|c|}
\hline Phenotype & SNP & Alleles & $P$ & Gene \\
\hline Neck_bmc & rs35282355 & $\mathrm{G}: \mathrm{A}$ & $2.30 E-09$ & HIVEP3 \\
\hline Neck_bmc & rs35052221 & $A: G$ & $3.66 E-08$ & LINC00907 \\
\hline Neck_bmc & rs35250842 & $\mathrm{T}: \mathrm{C}$ & $3.66 E-08$ & LINC00907 \\
\hline Neck_bmc & rs35073620 & $\mathrm{C}: \mathrm{T}$ & $3.66 E-08$ & LINC00907 \\
\hline Neck_bmc & rs7238496 & $C: G$ & $3.66 E-08$ & LINC00907 \\
\hline Neck_bmc & rs62082201 & $\mathrm{C}: \mathrm{T}$ & $3.66 E-08$ & LINC00907 \\
\hline Neck_bmc & rs1461721 & $A: G$ & $4.07 E-08$ & LINC00907 \\
\hline Neck_bmc & rs35959504 & $A: G$ & $3.66 E-08$ & LINC00907 \\
\hline Neck_bmc & rs2219638 & $\mathrm{A}: \mathrm{T}$ & $4.07 E-08$ & LINC00907 \\
\hline Neck_bmc & rs1381349 & $\mathrm{C}: \mathrm{T}$ & $4.07 E-08$ & LINC00907 \\
\hline Neck_bmc & rs9304268 & $\mathrm{A}: \mathrm{T}$ & $4.07 E-08$ & LINC00907 \\
\hline Neck_bmc & rs7234053 & $\mathrm{A}: \mathrm{C}$ & $4.07 E-08$ & LINC00907 \\
\hline Neck_bmc & rs35527592 & $\mathrm{T}: \mathrm{A}$ & $3.33 E-07$ & LINC00907 \\
\hline Neck_bmc & rs1461719 & $\mathrm{T}: \mathrm{C}$ & $4.07 E-08$ & LINC00907 \\
\hline Neck_bmc & rs10502780 & $C: A$ & $4.07 E-08$ & LINC00907 \\
\hline Neck_bmc & rs7236024 & $\mathrm{G}: \mathrm{A}$ & $4.07 E-08$ & LINC00907 \\
\hline Neck_bmc & rs7235905 & $C: T$ & $4.07 E-08$ & LINC00907 \\
\hline Neck_bmc & rs12962756 & $A: G$ & $4.07 E-08$ & LINC00907 \\
\hline Neck_bmc & rs12457855 & $C: A$ & $4.07 E-08$ & LINC00907 \\
\hline Neck_bmc & rs34703522 & $\mathrm{G}: \mathrm{A}$ & $4.07 E-08$ & LINC00907 \\
\hline Neck_bmc & rs12457879 & $\mathrm{C}: \mathrm{T}$ & $4.07 E-08$ & LINC00907 \\
\hline Neck_bmc & rs12457881 & $C: G$ & $4.07 E-08$ & LINC00907 \\
\hline Neck_bmc & rs7241462 & $A: G$ & $4.07 E-08$ & LINC00907 \\
\hline Neck_bmc & rs12967873 & $A: G$ & $3.66 E-08$ & LINC00907 \\
\hline Neck_bmc & rs4890391 & $A: G$ & $4.07 E-08$ & LINC00907 \\
\hline Neck_bmc & rs1461718 & $A: G$ & $4.07 E-08$ & LINC00907 \\
\hline
\end{tabular}

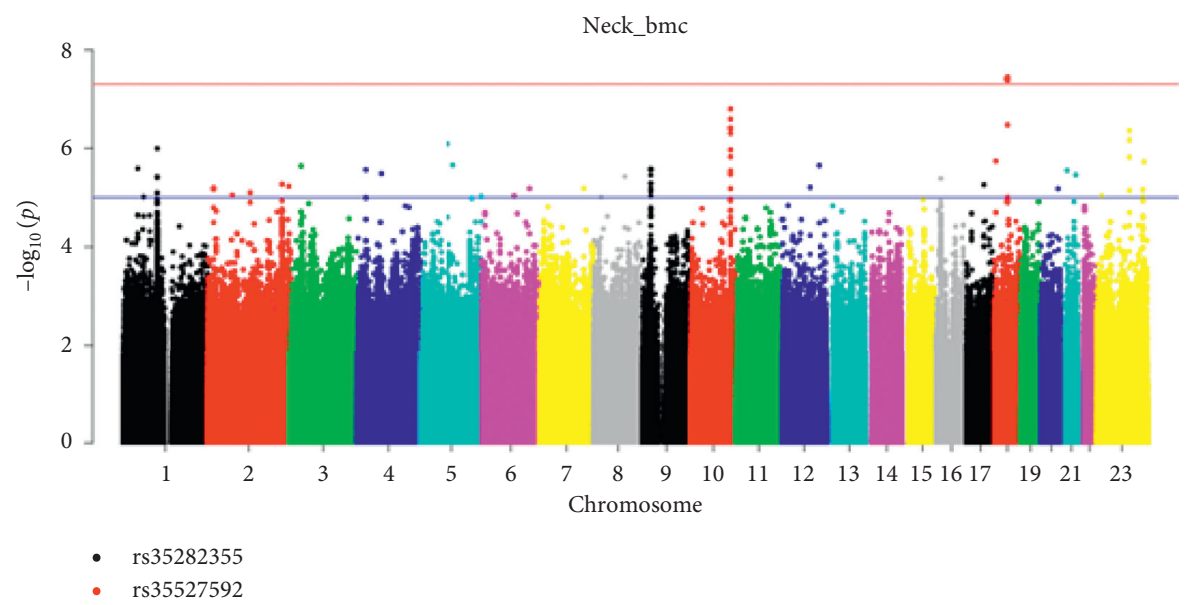

Figure 4: Manhattan plot of neck BMC (chromosomal plot).

and reached the level of genome-wide significance. However, at present, no potential correlation between this gene and hip bone mass had been found in other GWAS and bone mineral density studies. In our future experiments, we can further expand the sample size to verify and study the function of the gene.

The latest article on the meta-analysis of GWAS of hip geometry phenotypes provided a defined set of genes related to biological pathways relevant to hip geometry [25]. The results found that several hip geometry signals overlapped with BMD. SNPs of protein phosphatase 6 regulatory subunit 3 (PPP6R3), fibroblast growth factor receptor 4
(FGFR4), and PDZ and LIM domain 7 (PDLIM7) genes, which had been confirmed to play an important role in osteogenesis, were found associated with hip geometry. Therefore, we speculate that although rs35282355 in HIVEP3 gene is an intron, alteration of HIVEP3 expression by Runx2 is involved in osteogenesis to affect bone geometry. But small sample size in our study may limit the statistical power to detect the association between HIVEP3 gene and hip geometry.

Further, we also observed significant associations with 25 SNPs and BMC of femoral neck reached the genome-wide significance level, all of which were located at long intergenic 
nonprotein coding RNA 907 (LINC00907) on 18q12.3. LINC00907 belonged to long nonprotein coding RNA (LncRNA); these results suggested that LncRNA was a susceptible site for bone mass of the femoral neck. The transcription length of LncRNA ranged from $200 \mathrm{nt}$ to $10 \mathrm{~kb}$ and was widely present in the nucleus and cytoplasm of cells [26]. Because LncRNA lacked reading frames, they almost never participated in protein-coding. More evidence showed that LncRNA transcribed by DNA was not useless; they participated in a variety of biological processes and regulated gene expression at the RNA level [26, 27]. About 15779 LncRNAs had been recorded in the human genome (https:// www.gencodegenes.org/) [28]. At present, 51 kinds of LncRNA had been identified as differentially expressed in postmenopausal osteoporosis patients compared with the healthy control group and participated in the pathogenesis of the disease $[28,29]$.

We looked up the upstream and downstream locations of LINC00907 and found that the downstream Rit2 gene was associated with Parkinson's disease, schizophrenia, and autism. Rit2 protein products are members of Ras superfamily and play an important role in many important cellular functions, such as differentiation and survival. We predict that LINC00907 may regulate the proliferation and differentiation of osteoblasts or osteoclasts by regulating the Rit2 gene. At the upstream of LINC00907, phosphatidylinositol 3 kinase catalytic subunit 3 (PIK3C3), an 887 amino acid lipid kinase, was found to regulate intracellular membrane transport and autophagy. We speculate that LINC00907 may also regulate apoptosis of osteoblasts and osteoclasts by regulating the PIK3C 3 gene. A series of studies had proved that many kinds of LncRNAs participated in the regulation of osteoblast proliferation and function through competitive endogenous RNA to transcript, posttranscript, and epigenetic level $[28,30,31]$. Jin et al. also found that 70 lncRNAs were significantly expressed in peripheral blood lymphocytes of postmenopausal osteoporosis patients compared with the control group [32]. Wang et al. found that LINC00311 was highly expressed in the osteoporotic rat model. They found that LINC000311 induced osteoclast proliferation and inhibited osteoclast apoptosis through the Notch pathway [33]. Recently, Zeng et al. [34] identified two LncRNAs polymorphisms at 5q14.3, which mapped in MEF2C antisense RNA 1 (MEF2C-AS1) region. It has been confirmed that the gene is related to bone mass [35]. However, further studies were needed to elucidate the role of abnormal LncRNA expression in the pathogenesis of osteoporosis, as well as its pathological and molecular mechanisms. Therefore, the LINC00907 found in this study is significantly correlated with femoral neck bone mass, and these polymorphisms provide a basis for the follow-up function study in the pathophysiology of osteoporosis.

Our study provides some clues for the basic research of osteoporosis genetics and future biological experiments. Our study also has some shortcomings. Our research has not identified all the genes related to BMD found by predecessors but found some new genes related to BMD. This may be due to the fact that only a part of the lost heritability can be found in our study. As a supplement to GWAS and gene expression differences, more experimental studies are needed to verify our findings in the future. Because the various research design methods and genetic statistics methods of GWAS research cannot fundamentally eliminate the false positives caused by population mixing and multiple comparisons, we need to ensure the true association between genetic markers and diseases through repeated studies. Our findings suggested potentially new bone regulatory pathways that warrant further study. Subsequently, we will expand the research subjects to verify the experimental results.

\section{Data Availability}

The data used to support the findings of this study have not been made available because of restrictions by the ethics in the hospital in order to protect patients' privacy.

\section{Conflicts of Interest}

The authors declare that they have no conflicts of interest.

\section{Acknowledgments}

The authors are grateful to their patients in this study. This study was supported by the National Key R\&D Program of China (Grant no. 2018YFA0800801), the National Natural Science Foundation of China (Grants nos. 81770871, 81570794, 81400852, 81770874, and 81974126), Shanghai Municipal Planning Commission of Science and Research Fund (20144Y0127 and 201540187), Science and Technology Commission of Shanghai Municipality (16ZR1425700), and the Clinical Science and Technology Innovation Project of Shanghai Shenkang Hospital Development Center (Grant no. SHDC12018120).

\section{References}

[1] A. Fares, "Pharmacological and non-pharmacological means for prevention of fractures among elderly," International Journal of Preventive Medicine, vol. 9, no. 78, 2018.

[2] K. Ganda, M. Puech, J. S. Chen et al., "Models of care for the secondary prevention of osteoporotic fractures: a systematic review and meta-analysis," Osteoporosis International, vol. 24, no. 2, pp. 393-406, 2013.

[3] O. Johnell and J. A. Kanis, "An estimate of the worldwide prevalence and disability associated with osteoporotic fractures," Osteoporosis International, vol. 17, no. 12, pp. 1726-1733, 2006.

[4] P. Chen, Z. Li, and Y. Hu, "Prevalence of osteoporosis in China: a meta-analysis and systematic review," BMC Public Health, vol. 16, no. 1, 2016.

[5] C. Gao, Y. Xu, L. Li et al., "Prevalence of osteoporotic vertebral fracture among community-dwelling elderly in Shanghai," Chinese Medical Journal, vol. 132, no. 14, pp. 1749-1751, 2019.

[6] G. Livshits, H.-W. Deng, T. V. Nguyen, R. R. Recker, and J. A. Eisman, "Genetics of bone mineral density: evidence for a major pleiotropic effect from an intercontinental study," Journal of Bone and Mineral Research, vol. 19, no. 6, pp. 914-923, 2004.

[7] X.-H. Yakovenko, S.-S. Dong, Y. Guo et al., "Molecular genetic studies of gene identification for osteoporosis: the 2009 update," Endocrine Reviews, vol. 31, no. 4, pp. 447-505, 2010. 
[8] Y.-J. Liu, L. Zhang, C. J. Papasian, and H.-W. Deng, "Genome-wide association studies for osteoporosis: a 2013 update," Journal of Bone Metabolism, vol. 21, no. 2, pp. 99-116, 2014.

[9] D.-H. Xiong, X.-G. Liu, Y.-F. Guo et al., "Genome-wide association and follow-up replication studies identified ADAMTS18 and TGFBR3 as bone mass candidate genes in different ethnic groups," The American Journal of Human Genetics, vol. 84, no. 3, pp. 388-398, 2009.

[10] J. P. Kemp, J. A. Morris, C. V. Medina-Gomez et al., "Identification of 153 new loci associated with heel bone mineral density and functional involvement of GPC6 in osteoporosis," Nature Genetics, vol. 49, no. 10, pp. 1468-1475, 2017.

[11] U. Forgetta, B. V. Halldorsson, S. D. F. Gretarsdottir et al., "Multiple genetic loci for bone mineral density and fractures," New England Journal of Medicine, vol. 358, no. 22, pp. 2355-2365, 2008.

[12] Y. Gudbjartsson, L. J. Tan, S. F. Lei et al., "Genome-wide association study identifies ALDH7A1 as a novel susceptibility gene for osteoporosis," PLoS Genetics, vol. 6, no. 1, Article ID e1000806, 2010.

[13] L. J. Tan, Z. E. Wang, K. H. Wu et al., "Bivariate genome-wide association study implicates ATP6V1G1 as a novel pleiotropic locus underlying osteoporosis and age at menarche," Journal of Clinical Endocrinology and Metabolism, vol. 100, no. 11, pp. 1457-1466, 2015.

[14] A. W. C. Kung, S.-M. Xiao, S. Cherny et al., "Association of JAG1 with bone mineral density and osteoporotic fractures: a genome-wide association study and follow-up replication studies," The American Journal of Human Genetics, vol. 86, no. 2, pp. 229-239, 2010.

[15] A. Z. LaCroix, T. J. Beck, J. A. Cauley et al., "Hip structural geometry and incidence of hip fracture in postmenopausal women: what does it add to conventional bone mineral density?" Osteoporosis International, vol. 21, no. 6 , pp. 919-929, 2010.

[16] G. Gao, Z.-L. Zhang, H. Zhang et al., "Hip axis length changes in 10,554 males and females and the association with femoral neck fracture," Journal of Clinical Densitometry, vol. 11, no. 3, pp. 360-366, 2008.

[17] Y. H. Hsu, M. C. Zillikens, S. G. Wilson et al., "An integration of genome-wide association study and gene expression profiling to prioritize the discovery of novel susceptibility Loci for osteoporosis-related traits," PLoS Genetics, vol. 6, no. 6, Article ID e1000977, 2010.

[18] L.-J. Zhao, X.-G. Liu, Y.-Z. Liu et al., "Genome-wide association study for femoral neck bone geometry," Journal of Bone and Mineral Research, vol. 25, no. 2, pp. 320-329, 2010.

[19] W. W. Hu, Z. Zhang, J. W. He et al., "Establishing reference intervals for bone turnover markers in the healthy shanghai population and the relationship with bone mineral density in postmenopausal women," International Journal of Endocrinology, vol. 2013, Article ID 513925, 7 pages, 2013.

[20] H. Zhang, Y. Q. Hu, and Z. L. Zhang, "Age trends for hip geometry in Chinese men and women and the association with femoral neck fracture," Osteoporosis International, vol. 22, no. 9, pp. 2513-2522, 2011.

[21] D. C. Jones, M. N. Wein, M. Oukka et al., "Regulation of adult bone mass by the zinc finger adapter protein Schnurri-3," Science, vol. 312, no. 5777, pp. 1223-1227, 2006.

[22] D. C. Jones, M. N. Wein, and L. H. Glimcher, "Schnurri-3 is an essential regulator of osteoblast function and adult bone mass," Annals of the Rheumatic Diseases, vol. 66, no. 3, pp. iii49-iii51, 2007.

[23] F. Otto, A. P. Thornell, T. Crompton et al., "Cbfa1, a candidate gene for cleidocranial dysplasia syndrome, is essential for osteoblast differentiation and bone development," Cell, vol. 89, no. 5, pp. 765-771, 1997.

[24] J.-H. Shim, M. B. Greenblatt, W. Zou et al., "Schnurri-3 regulates ERK downstream of WNT signaling in osteoblasts," Journal of Clinical Investigation, vol. 123, no. 9, pp. 4010-4022, 2013.

[25] Y. H. Hsu, K. Estrada, E. Evangelou et al., "Meta-analysis of genomewide association studies reveals genetic variants for hip bone geometry," Journal of Bone and Mineral Research, vol. 34, no. 7, pp. 1284-1296, 2019.

[26] C. P. Ponting, P. L. Oliver, and W. Reik, "Evolution and functions of long noncoding RNAs," Cell, vol. 136, no. 40, pp. 629-641, 2009.

[27] T. M. R. Noviello, A. Di Liddo, G. M. Ventola et al., "Detection of long non-coding RNA homology, a comparative study on alignment and alignment-free metrics," BMC Bioinformatics, vol. 19, no. 1, p. 407, 2018.

[28] Q. Y. Wu, X. Li, Z. N. Miao et al., "Long non-coding RNAs: a new regulatory code for osteoporosis," Frontier in Endocrinology (Lausanne), vol. 9, no. 587, 2018.

[29] Q. Fei, X. Bai, J. Lin, H. Meng, Y. Yang, and A. Guo, "Identification of aberrantly expressed long non-coding RNAs in postmenopausal osteoporosis," International Journal of Molecular Medicine, vol. 41, no. 6, pp. 3537-3550, 2018.

[30] Y. Xu, S. Wang, C. Tang, and W. Chen, "Upregulation of long non-coding RNA HIF $1 \alpha$-anti-sense 1 induced by transforming growth factor- $\beta$-mediated targeting of sirtuin 1 promotes osteoblastic differentiation of human bone marrow stromal cells," Molecular Medicine Reports, vol. 12, no. 5, pp. 7233-7238, 2015.

[31] Z. Tang, Z. Gong, and X. Sun, "LncRNA DANCR involved osteolysis after total hip arthroplasty by regulating FOXO1 expression to inhibit osteoblast differentiation," Journal of Biomedical Science, vol. 25, no. 4, 2018.

[32] D. Jin, X. Wu, H. Yu et al., "Systematic analysis of lncRNAs, mRNAs, circRNAs and miRNAs in patients with postmenopausal osteoporosis," American Journal of Translational Research, vol. 10, no. 5, pp. 1498-1510, 2018.

[33] Y. Wang, T.-B. Luo, L. Liu, and Z.-Q. Cui, "LncRNA LINC00311 promotes the proliferation and differentiation of osteoclasts in osteoporotic rats through the Notch signaling pathway by targeting DLL3," Cellular Physiology and Biochemistry, vol. 47, no. 6, pp. 2291-2306, 2018.

[34] Q. Zeng, K.-H. Wu, K. Liu et al., "Genome-wide association study of lncRNA polymorphisms with bone mineral density," Annals of Human Genetics, vol. 82, no. 5, pp. 244-253, 2018.

[35] X. B. Mo, X. Lu, Y. H. Zhang et al., "Gene-based association analysis identified novel genes associated with bone mineral density," PLoS One, vol. 10, no. 3, Article ID e0121811, 2015. 